\title{
DEVELOPMENT OF 3D WOVEN FABRIC BASED PRESSURE SWITCH
}

\author{
Khubab Shaker ${ }^{1}$, Yasir Nawab ${ }^{\star 1}$, Muhammad Usman Javaid ${ }^{1,2}$, Muhammad Umair $^{1}$, Muhammad Maqsood ${ }^{1}$ \\ ${ }^{1}$ Department of Weaving, Faculty of Engineering and Technology, National Textile University, Faisalabad, Pakistan \\ ${ }^{2}$ Department of Textile Materials, Faculty of Textile, Technical University of Liberec, Czech Republic \\ ${ }^{*}$ Corresponding author: Yasir Nawab, e-mail: yasir.nawab@yahoo.com
}

\begin{abstract}
:
This paper introduces a 3D woven fabric-based approach for the development of pressure switch. A fabric substrate, being elastic and extendable is very useful in addition to its high breaking strength and low cost. The developed resistive-type switch is based on the multilayer interlock 3D fabrics. In the top and bottom layers, certain number of conductive yarns are woven separated by cotton yarns in both transversal and thickness direction. Application of pressure makes the layers of conductive yarn to come in contact, resulting in a short circuit, which may be recorded using multi-meters. Removing the pressure cause the connection points to separate away and it depends on the weave design. Such switch can be used as an on/off switch for usage in security systems, can be sewn into carpets and wearable garments for a number of purposes.
\end{abstract}

\section{Keywords:}

textile pressure switch, 3D multilayer fabrics, conductive yarns

\section{Introduction}

Interactive textiles are the fibrous materials having sensing and actuating properties, detecting change in the environment, mechanical or electrical conditions [1]. They are a unique combination of textiles and electronics having three components to sense, actuate and control. The textile structure being elastic and extendable acts as perfect substrate, giving more breaking strength and a lower cost. The concept is best suited for wearable electronic textile sensors, used to improve safety and comfort properties, providing communication and entertainment in countless other ways. Flexible plastic optical fibres are also used in the woven textile-based pressure sensors [2].

The textile-based resistive pressure sensors make use of metallic yarns, placed at different positions such that the application of external pressure brings them to contact and helps to decrease the resistance. The sensing fabric forms the relationship between pressure to be measured and resistance. It is more efficient due to simple, easy-to-make structure and faster measurement than capacitive sensors [3].

Wearable sensors have diagnostic, as well as monitoring applications [4]. They are widely used in functional apparels, shoe designing, construction, automobiles, medical and sport science, fitness and health, research on human biomechanics and ergonomics, security and protection. Some typical functions of such sensors include on/off function, as well as adjustable lighting or sound. A switch incorporated into a sofa, chair, carpet or wall covering allows the user to turn on and adjust a lighting or stereo system, or to control a climate or security system. The textile-based pressure sensors provide the comfort and wearability properties [5].
A lot of research on smart textiles is being carried out around the globe, because of their versatility in use. Highly durable and flexible multilayer electronic circuits are constructed on textile substrates by electronic embroidery, using conductive yarns [6]. Pressure sensors based on resistive sensors use compressive materials, employing two or more layers. Such arrangements increase the complexity, thickness, weight, and cost of the sensors with some other disadvantages. It is important to design a recoverable structure so that the contacting points can be disconnected after the removal of pressure [7].

The task of integrating or fitting electrical and electronic circuit within clothing presents a number of problems too, including the incorporation of switches. The use of a textile switch may be a possible solution. The textile form flexible resistive element and flexible conductive materials, connective to external circuitry are used [8]. The textile switch comprises of an arrangement of two electrically conductive contact portions separated by resilient means such as compressible foam material [9]. The application of a sufficient force causes the compressible foam material to yield allowing the conductive components to come in contact. Removal of the applied force allows the foam material to return to its shape, separating the both layers.

The aim of this research is to develop a textile switch using 3D multilayer fabric structure having conductive and insulating yarns, by means of weaving. A multilayer fabric consists of two or more layers, linked to one another at fastening points. The numbering of layers is done consecutively from top to bottom. Multilayer interlock structures are either orthogonal or angle interlock [10]. Multilayer concept involves stitching and separation of woven fabric on loom [11] in localised areas, making it easy to develop pressure switch. The objective is related to simple and basic application, that is, just to start and stop the circuit. 


\section{Experimental}

The materials used in the development of pressure switch include cotton and multifilament steel yarns. The cotton yarns were used to form the insulating layer, while multifilament steel yarns were used for the conductive layer. The steel yarns provided required amount of conduction, while cotton was used in coarser count to achieve the separation among the conductive yarn.
Five 3D multilayer interlock woven samples were produced having different weaves and structure as mentioned in Table 1. Samples were made with number of layer varying from one to three, and the interlacement pattern (weave design) of yarns in all the samples was different as shown in Figure 1. Weave design is the determining factor for the possibility of contact between the conductive yarns. Objective of different weaves and number of layers was to investigate the performance of different structures and to find the most efficient pressure switch.

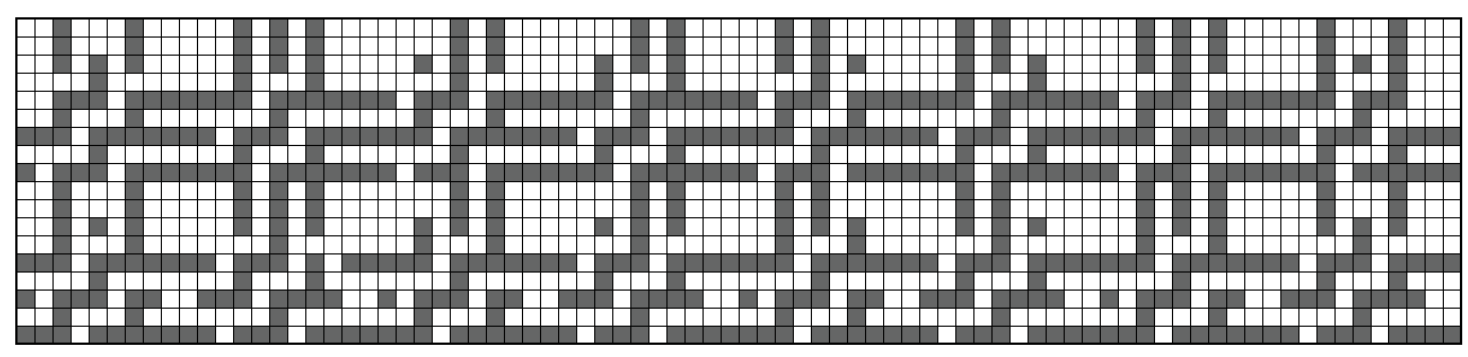

W1

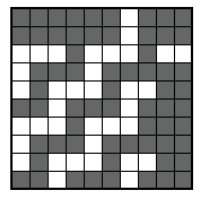

W2

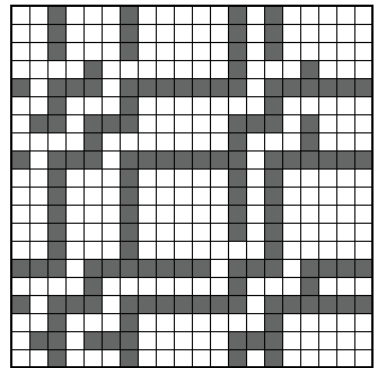

W3

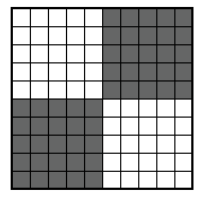

W4

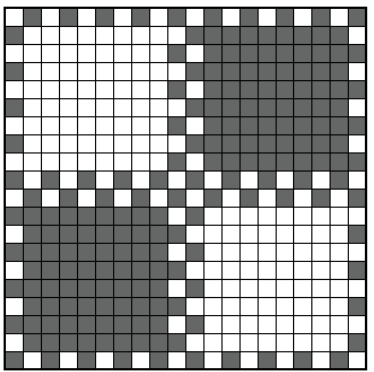

W5

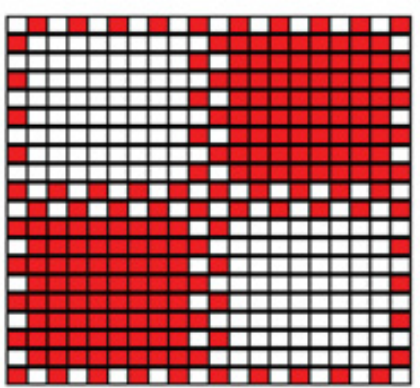

(a)

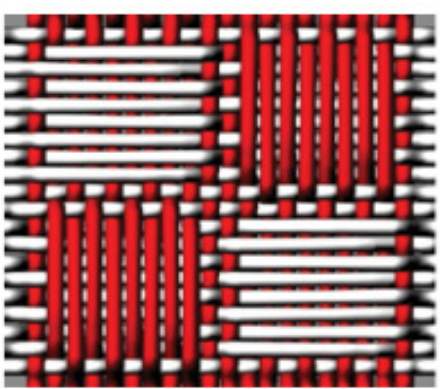

(b)

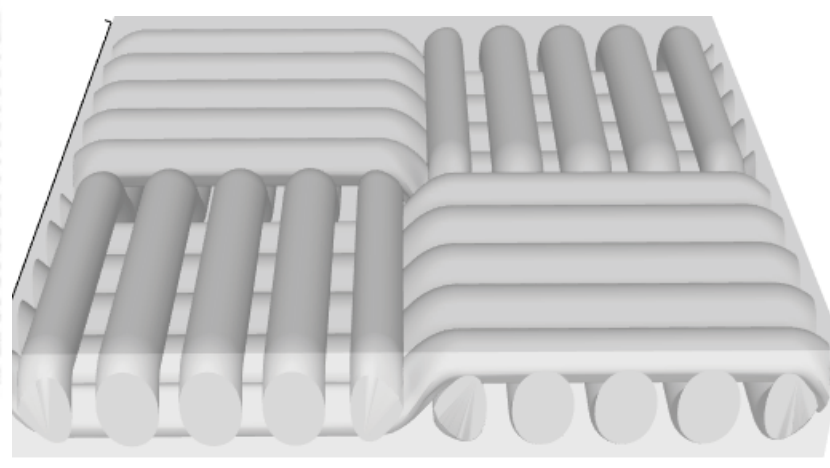

W4

W5

Figure 1.The weave designs of all samples, cross-sectional view of W4 and 2D view of W5

Table1. Structural comparison of samples

\begin{tabular}{|c|c|c|c|c|}
\hline Sample & No. of layers & Warp distribution & Weft distribution & Weave design \\
\hline 1 & Two & $8 \mathrm{C}+2 \mathrm{~S}$ & $8 \mathrm{C}+2 \mathrm{~S}$ & W2 \\
\hline 2 & Three & $8 \mathrm{C}+2 \mathrm{~S}$ & $8 \mathrm{C}+2 \mathrm{~S}$ & W3 \\
\hline 3 & Three & $8 \mathrm{C}+2 \mathrm{~S}$ & $8 \mathrm{C}+2 \mathrm{~S}$ & W4 \\
\hline 4 & Single & $4 \mathrm{C}+1 \mathrm{~S}$ & $4 \mathrm{C}+1 \mathrm{~S}$ & W5 \\
\hline 5 & Single & $4 \mathrm{C}+1 \mathrm{~S}$ & $4 \mathrm{C}+1 \mathrm{~S}$ & \\
\hline
\end{tabular}

Where, C: cotton yarn and S: steel yarn 
All the samples were produced on shuttle dobby loom. The W1 was a double-layered structure, having repeat of 10 ends and 10 picks. The sequence of cotton and steel yarns in warp and weft was 8 and 2, respectively. The final structure had warp yarns arranged in such a way to give a float to conductive yarn, and bringing it into contact only at the required point.

W2 was a three-layered structure, having free yarns in middle layer, serving the function of an insulator. The W3 was a derivative of $\mathrm{W} 2$, also produced in three layers, but middle layer having some bindings instead of free yarns, allowing conductive yarns to come in contact at the binding points. These bindings provide the required tension so that conductive yarns can come in contact and separate by the application and release of pressure.

The W4 and W5 were single layered, having matt weave with every fifth warp and weft thread being conductive. The only difference between W4 and W5 was the stitching ends, induced in a way similar to BFC design. The W4 was produced on two frames, while W5 on four frames due to slight variation of design. The third and fourth frames of W5, producing plain weave were used to provide tension to the threads making the fabric structure stable.

\section{Results and discussion}

There were two major requirements for the multilayer woven interlock structures to work as switch. First was the establishment of a contact point between the conductive yarns of the top layer and the bottom layer, upon application of pressure. The other requirement was the ability of structure to separate the layers on removing pressure, causing the conductive yarns to separate. The fulfilment of these requirements was checked by resistivity test.

\section{Resistivity test}

Design of the multilayer woven interlock structure renders the conductive yarn to be woven in two types of connections: series or parallel. The conductance of series connection is checked using two conductive yarns in the top and bottom layers, one for positive(+ve) terminal and the other for negative $(-\mathrm{ve})$ terminal of digital voltmeter as shown in Figure 2. To check the conductivity for the parallel circuit, it is required to connect some conductive yarns to positive terminal and some to negative terminal of digital voltmeter and then joined to get different contact points.

Firstly, the conductivity and resistivity of both series and parallel connections was checked with the same number of contact points using two conductive yarns representing the top and bottom layers of the switch. The conductance was checked such that both the yarns get joined at only one point and then after every increment in point of contact, at a constant current of $1 \mathrm{amp}$. The results in Table 2 showed that reduction in the resistivity for parallel connection is more than that for series connections, as the number of contact points increase. Then, graph was plotted between point of contacts (x-axis) and resistivity (y-axis) for both of the connections as shown in Figure 3.

The resistivity test was performed for all the multilayer woven interlock structures, applying some pressure. Three different dead weights, 30, 40 and $50 \mathrm{~g}$ were used to exert force on the top of switch. Contact was observed at an optimum weight of 40 g. Three samples of each type were produced, and resistivity test on each sample was performed three times to ensure the authenticity of results.

The upper and lower layers of W1 had warp floats at the back, producing some up-points for connection where conductive

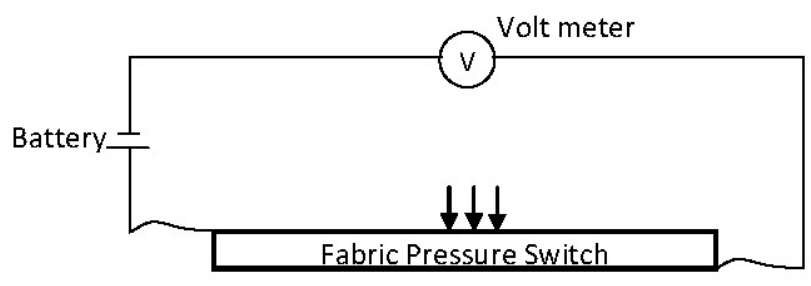

Figure 2. Testing schematic diagram

Table 2.Comparison between conductance and resistivity of series and parallel connections

\begin{tabular}{|c|c|c|c|c|}
\hline \multirow{2}{*}{ Points of contact } & \multicolumn{2}{|c|}{ Conductance (volt) } & \multicolumn{2}{c|}{ Resistivity (ohm) } \\
\cline { 2 - 5 } & Series & Parallel & Series & 33 \\
\hline 1 & 0.011 & 0.030 & 88 & 18 \\
\hline 2 & 0.012 & 0.056 & 84 & 15 \\
\hline 3 & 0.015 & 0.067 & 68 & 13 \\
\hline 4 & 0.016 & 0.077 & 63 & 12 \\
\hline 5 & 0.017 & 0.083 & 60 & 12 \\
\hline
\end{tabular}




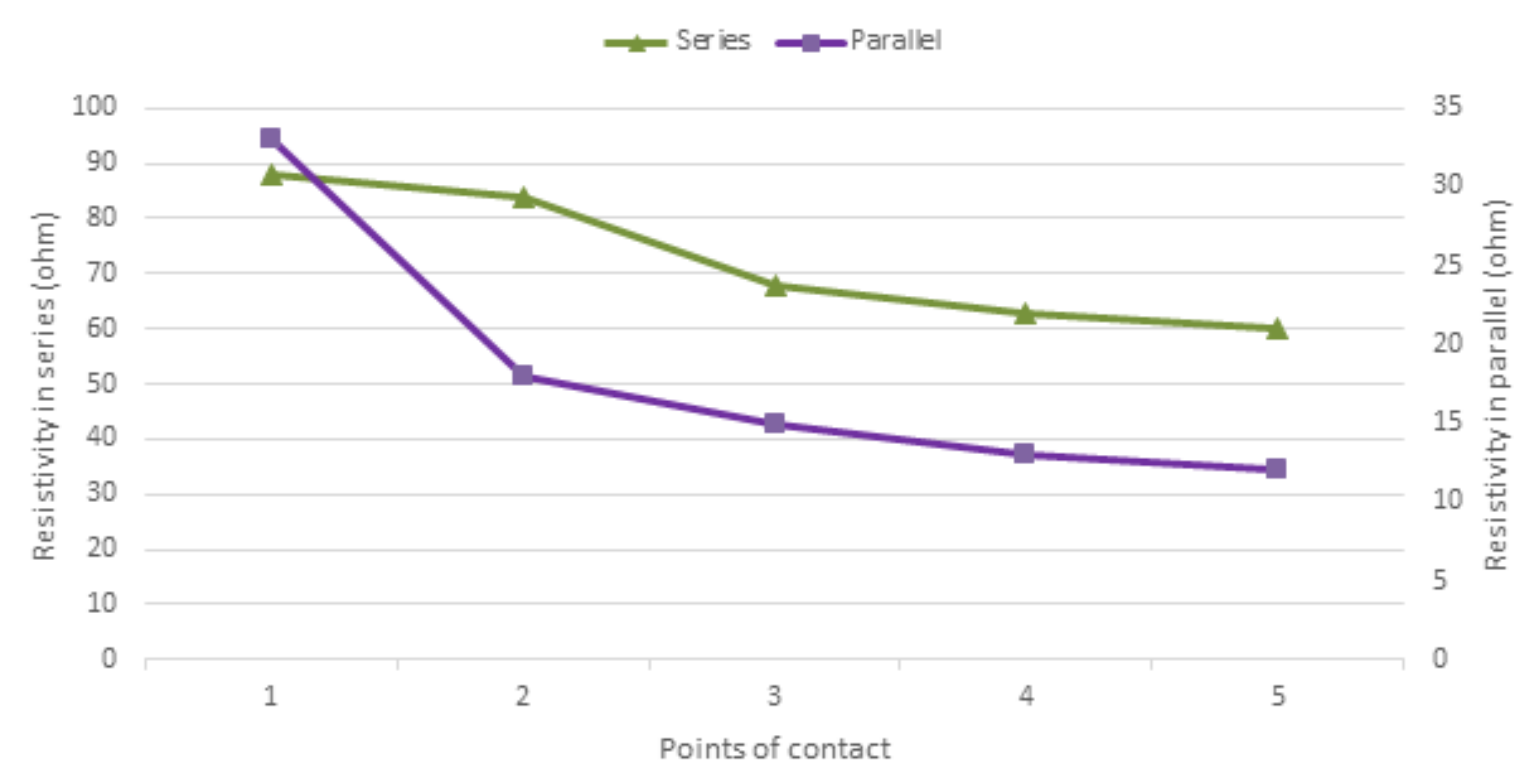

Figure 3. Comparison for the resistivity of series and parallel connections

yarn is visible. It was expected that on the application of pressure, warp floats of upper layer will connect with the uppoints of lower layer; thus completing the circuit, and remains separate in normal condition due to higher thread density. But in the resistivity testing, no conductance was observed, showing the absence of any contact points between the upper and lower layer. No meeting points between conductive yarns of both layers resulted in failure W1 as switch.

The W2 and W3 were both three-layered structures, the top and bottom layers having conductive yarns, while the middle layer was insulating. In case of W2, there were free threads in the middle layer, having longer floats without any interlacements, while W3 had some intersections in the middle layer. In the resistivity test, W2 worked like a textile switch on some points but not on all points. The space between free threads of the middle layer allowed some conductive yarns of the top and bottom layers to come in contact, and blocking others due to insulating effect, thus making the effect random. The middle layer of W3 had some intersections, so there were no meeting points between upper and lower layers and no conduction was observed.

The structures W4 and W5 were single-layered having basket weave, with a slight variation. Due to single-layered structure, there was no insulation between the conductive yarns of warp and weft. The W4 had a lower yarn density, so the resulting yarns were much slack in the structure and the conductive yarns were always in contact. So, the W4 failed due to nonfulfilment of the second requirement. The plain woven threads were introduced in the structure of W4 to get the structure W5. The aim was to avoid the slackness of yarns and help in the restoration of original structure on removal of pressure. Despite the plain woven threads used to maintain the require tension, W5 was also not working as a switch as its circuit was short.
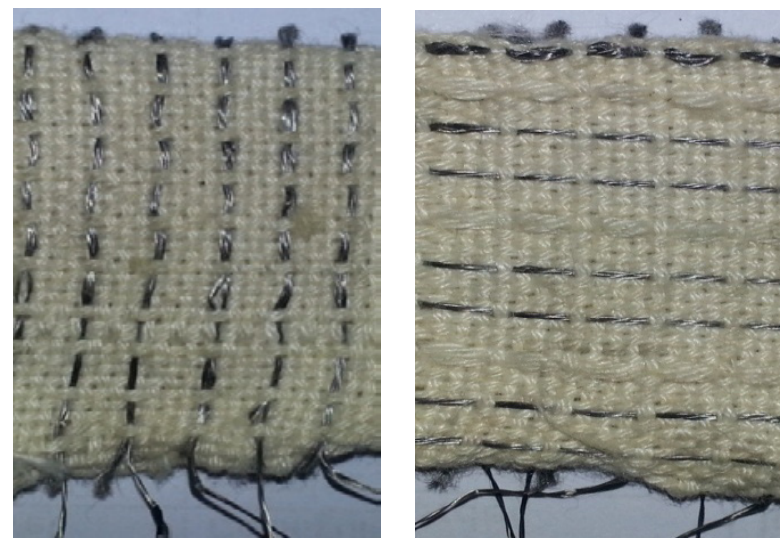

Figure 4. Sample of multilayer interlock woven structure, W2

\section{CONCLUSIONS}

The results of these structures showed that multilayer interlock woven fabrics containing conductive yarns in the top and bottom layers can be employed as pressure switch. The best results were achieved by three-layered woven structure with the design W2. The important factor for the structure to work as switch is the sequence of cotton and conductive yarns in warp and weft. The other thing to be considered is the contact between conductive yarns of both the layers and the ability of structure to recover its shape, that is, to detach the both layers. The main advantages of such type of pressure switch are their capability to absorb pressure, flexibility, high breaking strength and low cost.

\section{References}

[1] Leitch, P., \& Tassinari, T. H. (2000). Interactive Textiles: New Materials in the New Millennium. Part 1. Journal of Industrial Textiles, 29(3), 173

[2] Rothmaier, M., Luong, M. P., \& Clemens, F. (2008). Textile Pressure Sensor Made of Flexible Plastic Optical Fibers. Sensors, 8(7), 4318-4329. 
[3] Meyer, J. (2008). Textile Pressure Sensor: Design, Error Modeling and Evaluation. ETH.

[4] Patel, S., Park, H., Bonato, P., Chan, L., \& Rodgers, M. (2012). A review of wearable sensors and systems with application in rehabilitation. Journal of Neuroengineering and Rehabilitation, 9(1), 21

[5] Dunne, L. E., Brady, S., Smyth, B., \& Diamond, D. (2005). Initial development and testing of a novel foambased pressure sensor for wearable sensing. Journal of Neuroengineering and Rehabilitation, 2(1), 4.

[6] Post, E. R., Orth, M., Russo, P. R., \& Gershenfeld, N. (2000). E-broidery: Design and fabrication of textile-based computing. IBM Systems Journal, 39(3.4), 840-860.
[7] Tao, X., \& Zhang, H. (2006). Pressure sensing fabric. United States.

[8] Lussey, D., Jones, D., \& Leftly, S. (2007). Flexible switching device. US 7301435 B2.

[9] Farringdon, J. (2003). Electrical switch for use in garments. US 6642467 B2.

[10] Nawab, Y., Legrand X., \& Koncar V. (2012). Study of changes in 3D-woven multilayer interlock fabric preforms while forming. Journal of the Textile Institute, 103 (12), 1273.

[11] Grosicki, Z., \& Watson, W. (1977). Watson's advanced textile design: Compound woven structures. NewnesButterworths. 